\title{
NOTAS PARA A HISTÓRIA DA PSICOLOGIA DA SAÚDE
}

\author{
Cassiano Ricardo Rumin \\ Psicólogo, especialista em Saúde Pública (FCF/UNESP), mestre em Ciências Médicas (FMRP/USP), \\ docente e coordenador da Pós-Graduação lato sensu em Psicologia da Saúde (Faculdades \\ Adamantinenses Integradas - FAI)
}

\begin{abstract}
Resumo
A história da Psicologia da Saúde pode ser abordada pela necessidade dos serviços hospitalares compreenderem elementos psicodinâmicos oriundos das condições de adoecer e dos processos de cuidado. Mais tarde, o direcionamento das práticas em Psicologia da Saúde para a atenção primária ampliou o conjunto de conhecimentos e de referências de atuação dos profissionais de psicologia. Esta história segue ampliando suas áreas de abrangência e passando por redefinições derivadas de processos reflexivos das entidades de formação profissional e de políticas públicas. Objetivou-se neste artigo caracterizar componentes teóricos e técnicos que se apresentam no momento de comemoração dos 50 anos de reconhecimento da profissão no Brasil. Os resultados indicam a ascendente participação da profissão nas políticas públicas sociais e a retomada de práticas dirigidas a comunidade que experimentaram retrocessos. Conclui-se afirmando que a posição ocupada pela profissão nas políticas de saúde coletiva são movimentos de afirmação de direitos que historicamente foram enfraquecidos.
\end{abstract}

Palavras-chave: psicologia da saúde; história da psicologia; políticas públicas.

\section{NOTES FOR THE HISTORY OF HEALTH PSYCHOLOGY}

\begin{abstract}
The history of Health Psychology can be addressed from the perspective of the need for hospital services to understand psychodynamic elements arising from illness conditions and care processes. Later, the orientation of Health Psychology practices to primary health care expanded the set of knowledge and references for psychology professionals' activities. This history continues to broaden its coverage areas and goes through redefinitions, deriving from reflexive processes of professional education entities and public policies. The aim in this paper was to characterize theoretical and technical components at a time when the 50th anniversary of the profession's recognition in Brazil is celebrated. The results indicate the increasing participation of the profession in social public policies and the recovery of community-oriented practices that experienced backlashes. In conclusion, it is affirmed that the position the profession occupies in collective health, are movements to affirm historically weakened rights.
\end{abstract}

Keywords: health psychology; history of psychology; public policies. 


\title{
NOTAS PARA LA HISTORIA DE LA PSICOLOGÍA DE LA SALUD
}

\begin{abstract}
Resumen
La historia de la Psicología de la Salud puede ser tratada por la necesidad de que los servicios hospitalarios comprendan elementos psicodinámicos oriundos de condiciones de adolecer y procesos de cuidado. Después, el direccionamiento de las prácticas en Psicología de la Salud hacia la atención primaria amplió el conjunto de conocimientos y referencias de actuación de los profesionales de psicología. Esta historia sigue ampliando sus áreas de alcance y pasando por redefiniciones derivadas de procesos reflexivos de las entidades de formación profesional y de políticas públicas. La finalidad en este artículo fue caracterizar componentes teóricos y técnicos que se presentan en el momento de conmemoración de los 50 años de reconocimiento de la profesión en Brasil. Los resultados indican la ascendente participación de la profesión en las políticas públicas sociales y la retomada de prácticas dirigidas a la comunidad que experimentaron retrocesos. Se concluye afirmando que la posición ocupada por la profesión en las políticas de salud colectiva son movimientos de afirmación de derechos que históricamente fueron debilitados.
\end{abstract}

Palabras-clave: psicología de la salud; historia de la psicología; políticas públicas.

\section{INTRODUÇÃO}

Ao longo da história da Psicologia a perspectiva de desenvolver-se em áreas ligadas aos cuidados em saúde estabeleceu um cenário amplo de atuações. Particularmente no Brasil, a Psicologia da Saúde foi formulada no contexto da integralidade das ações em saúde, mesmo com os entraves a efetivação de tal proposição (Spink, 2007).

É possível visualizar a articulação histórica da Psicologia ao cenário dos cuidados em saúde, a partir do emprego de construtos em Psicologia por outras profissões de saúde. Grilo e Pedro (2005) reconhecem ao menos quatro destas contribuições: na promoção da saúde, na atenção a reação relativa ao diagnóstico da doença, na pediatria e na relação do profissional de saúde com o indivíduo adoecido.

Por esta via, pesquisas em Psicologia começaram a se associar as práticas de reabilitação em saúde. Intervenções historicamente relevantes e, ainda atuais, ligadas a reabilitação e Psicologia são aquelas dirigidas à atenção de veteranos de guerra. Dunn, Julian, Formolo, Green e Chicoine (2011) abordam a presença de dores nas costas e no pescoço e sua inter-relação com o transtorno de estresse pós-traumático. Outro exemplo onde a dor crônica é um elemento de destaque nas investigações entre Psicologia e reabilitação refere-se a busca por evidências entre caracteres de personalidade e a manifestação da fibromialgia (Pérez-Pareja, Sesé, González-Ordi \& Palmer, 2010). As características de personalidade e a articulação com um processo de adoecimento também são 
abordadas nas dermatites (Gascon et al. 2012). Com o desenvolvimento das técnicas de transplante de órgãos as investigações em Psicologia também tiveram destaque nesta área de atenção à saúde (Jin et al. 2012).

Posteriormente, a constituição de um corpo teórico/técnico que estruturaria a intervenção nos processos de saúde/doença pôde ser reconhecida nas proposições da Psicologia da Saúde. Na América Latina esta se amplia em direção "a las cuatro dimensiones de atención de la salud humana: promoción y educación, prevención, tratamiento y rehabilitación y/o cuidados paliativos" (Sebastiani \& Maia, 2006 p.05). Flórez-Alacón (2006) destacam que na Colômbia, a Psicologia da Saúde se diferenciou das práticas de Medicina Comportamental desenvolvidas na década de 1970 e se especializou na Psicologia Clínica da Saúde, com ênfase nas atividades de prevenção secundária e terciária. Entretanto, enfatiza que a atuação de 25 centros colombianos de pesquisa em Psicologia da Saúde necessita articular as investigações e as intervenções às práticas em saúde pública como o caminho de evolução deste campo de conhecimento.

O desenvolvimento da Psicologia da Saúde no Brasil também se articula as perspectivas da Psicologia Clínica da Saúde, onde a população abordada guarda em comum a presença de um agravo à saúde. Estas referências de atuação são fundamentadas na noção de que:

"O que distingue o campo da Psicologia da Saúde de outros campos da Psicologia é o fato que os indivíduos aqui atendidos têm, em geral, um problema ligado à sua saúde física, de diversas ordens ou gravidades possíveis. Usualmente, trata-se de um indivíduo que tem um problema orgânico relacionado a aspectos comportamentais ou emocionais, podendo tanto o problema orgânico quanto os aspectos comportamentais/emocionais serem causa ou conseqüência da relação" (Gorayeb, 2010 p.119).

Ao entrelaçar o termo Psicologia da Saúde à existência de um agravo aos sistemas orgânicos, o autor ressalta não restringir-se a Psicologia Hospitalar. Remete o leitor aos três níveis de atenção à saúde, preconizados pelo Sistema Único de Saúde. Uma sólida referência da atenção em Psicologia da Saúde nos distintos níveis de atenção pode ser apreciada na leitura de Miyazaki et al. (2002). A Psicologia Hospitalar estaria posicionada em um campo da atenção em saúde:

\footnotetext{
"a atenção primária, que se dá nos postos de saúde com o intuito de tratar alterações do estado de saúde mais simples; a atenção secundária, que se dá já em um nível de especialidade, em geral em situação ambulatorial; e a atenção terciária, que é prestada em
} 
hospitais. O nível de atenção terciária em Psicologia poderia ser o que se convencionou no Brasil chamar de Psicologia Hospitalar" (Gorayeb, 2010 p.120).

Castro e Bornholdt (2004, p.48) enfatizam o pertencimento da Psicologia Hospitalar a área de conhecimento denominada Psicologia da Saúde. Afirmam que a citada área de conhecimento utiliza saberes interdisciplinares e referenciais da Psicologia Clínica e Comunitária para intervir em distintos contextos. Os conhecimentos interdisciplinares são instrumentos para as "atividades coletivas de promoção e prevenção em saúde" (Silveira \& Vieira, 2009). Os conhecimentos originados na Psicologia Clínica tem articulação imediata com os estudos de fenômenos psicossomáticos (Ávila, 2004; Ávila, 2006). Já a Psicologia Comunitária envolve uma perspectiva de observação e compreensão das necessidades, onde o sofrimento singular do indivíduo que integra uma comunidade vincula-se ao sofrimento coletivo, na medida em que muitos integrantes são envolvidos. A abrangência aumentada da intervenção clínica comunitária conserva "uma escuta dialógica e hermenêutica, com a utilização de técnicas ativas e uma interpretação de situações-problema do ponto de vista individual, grupal e sócio-comunitário. A resolução de conflitos passa do âmbito pessoal para a criação conjunta de soluções" (Nery \& Costa, 2008 p.243).

Ao descrever os conhecimentos empregados em Psicologia da Saúde, Silva (2002) destaca que devem estar centrados no conceito de Saúde Integral, numa "aproximação positiva da saúde e claramente implica ações para a promoção, prevenção e tratamento" (SILVA, 2002 p.28). A perspectiva positiva enfoca os fatores de proteção à saúde, aquilo que transforma ou melhora as respostas aos fatores envolvidos no processo de saúde-doença. Analisam-se a resiliência, as habilidades interpessoais, a esperança, a criatividade e outros fatores protetivos à saúde (Calvetti; Muller \& Nunes, 2007). Estudos sobre a religiosidade também se agregam à perspectiva positiva em saúde, tal como observado em Alves et al. (2010).

\section{PSICOLOGIA DA SAÚDE E SEUS DETERMINANTES HISTÓRICOS}

Até agora foram discutidos parâmetros conceituais para a definição da Psicologia de Saúde. Tais fatos não aconteceram desatrelados de determinantes históricos que, em especial no Brasil, imprimiram caracteres particularizados a história da Psicologia brasileira. A redemocratização e a reforma sanitária 
incentivaram alguns modelos da Psicologia. Há destaque para as concepções da Psico-Higiene que conforme destacado por Guirado (2004) tem como principal característica, organizar intervenções em saúde mental que abranjam os espaços relacionais dos sujeitos: a família, a escola, o trabalho e as atividades comunitárias. Vale salientar que o emprego desta concepção pode guardar a noção de "adaptação do indivíduo privado ao público" (Bernardes e Guareschi, 2010 p.273) e a partir de uma racionalidade técnica estabelecer um esquadrinhamento social. Para as autoras:

\begin{abstract}
"as práticas psicológicas, ao tornarem uma evidência as diversas formas de vida, acabam por incitar uma racionalidade de direito específica a cada taxionomia social: programas voltados para a saúde da mulher, desdobrados em gestantes, adolescentes gestantes, vítimas de violência doméstica, mulheres oncológicas, etc.; programas voltados para DST/AIDS, desdobrados em homossexuais, drogaditos, mulheres casadas, adolescentes, prostitutas, etc.; programas voltados para educação em saúde, desdobrados em coleta de lixo, reciclagem de lixo, utilização da água, utilização de medicamentos, cuidados com a alimentação, etc" (Bernardes e Guareschi, 2010 p.275).
\end{abstract}

A urgência em estabelecer ações políticas e de orientação comunitária como respostas às crises ditatoriais e políticas das décadas de 1970 a 1990 (Scarparo e Guareschi, 2007) mobilizaram outros condicionantes componentes da Psicologia da Saúde. Passou-se a identificar a comunidade, na concepção de Jovchelovitch (2008, p.130), "como um importante nível de análise no campo da saúde" que envolveria a concepção do adoecer e do sofrimento fomentados pela opressão e violação de direitos. A ditadura militar é responsável por uma ameaça que se estende aos vínculos intersubjetivos constituídos na comunidade. Conforme destaca Käes (2005) o Estado - responsável pela proteção dos indivíduos - investe contra a comunidade produzindo ações violadoras de direitos e solapando as "formações intermediárias". Estas sustentariam "a vida psíquica e os vínculos intersubjetivos e sociais" (Käes, 2005 p.172); ataques dirigidos a elas incitariam a atomização do sofrimento, negando o reconhecimento do sofrer no espaço comunitário.

Estes condicionantes alavancaram a aproximação com as comunidades, visando suprir as necessidades de saúde da população, utilizando a rua e o domicilio como espaços de intervenção (Sousa e Cury, 2009). O Teatro do Oprimido e o Teatro Jornal (Boal, 1975) cumpriram a função de ocupar a rua e espaços públicos na América Latina abalada pelo militarismo. Santos e Kirschbaum (2008) afirmam que o domicílio passou a ser tomado como espaço 
de intervenção por outros profissionais de saúde desde 1920. Já para os profissionais de Psicologia o domicílio fortaleceu-se como espaço de intervenção, com as práticas de acolhimento que se seguiram às reorganizações da rede básica de saúde com a viabilidade de projetos em prol da saúde da família (Soares, 2005). As reorganizações da rede básica se efetivaram:

\begin{abstract}
"por meio do exercício de práticas gerenciais e sanitárias democráticas e participativas, sob forma de trabalho em equipe, dirigidas a populações de territórios bem delimitados, pelas quais assume a responsabilidade sanitária, considerando a dinamicidade existente no território em que vivem essas populações (Brasil, 2006 p. 2).
\end{abstract}

As práticas gerenciais servem de incentivo ao desenvolvimento de pesquisas que fundamentem as evidências empíricas relativas à eficiência das intervenções em Psicologia da Saúde (Gidron, 2002). Já o exercício democrático estimulou concepções teóricas como a Psicologia Social da Saúde, a se expressar no cenário brasileiro. Spink (2003) afirma que a Psicologia Social da Saúde está edificada a partir da efetivação de direitos sociais, da cidadania e compreensão coletiva dos processos de saúde/doença. Tal orientação determinou à prática do profissional em Psicologia uma atuação que:

\begin{abstract}
"se dá principalmente nos serviços de atenção primária à saúde, focaliza a prevenção da doença e a promoção da saúde e incentiva os atores sociais envolvidos para a geração de propostas de transformação do ambiente em que vivem. Trata-se, portanto de um processo de transformação crítica e democrática que potencializa e fortalece a qualidade de vida" (Camargo-Borges \& Cardoso, 2005 p. 29).
\end{abstract}

Tanto a Psicologia da Saúde baseada em evidências quanto a Psicologia Social da Saúde atenderiam as proposições da Associação Brasileira de Ensino em Psicologia (ABEP), que enfatiza a presença qualificada no SUS. Para isto, é necessária a participação do profissional de Psicologia em processos formativos compartilhados "com todos os profissionais do campo [saúde] e com novas exigências que ultrapassam o papel tradicional do psicólogo" (ABEP, 2006 p.15).

A presença qualificada no SUS também envolve a atenção à saúde mental (Silveira e Vieira, 2009) que se configura nas unidades públicas de saúde. Bittencourt \& Mateus (2006, p.331) destacam que a inclusão das práticas de Saúde Mental na atenção básica "passa por importantes transformações conceituais e operacionais, reorientando-se o modelo historicamente centrado na referência hospitalar por um novo modelo de atenção descentralizado e de base comunitária". De acordo com Guareschi et al. (2009 p.43): 
"O SUS visibiliza a concepção de saúde pautada não mais na simples ausência de doença, mas na promoção das condições de vida dos sujeitos, lançando assim um olhar integral que abarque os diversos contextos sociais e culturais em que os indivíduos estão inseridos, o que caracteriza a saúde coletiva".

A proposição exposta acima pelas autoras é o ponto de aproximação entre a Psicologia da Saúde e as políticas sociais. De acordo com Perez, Constantino e Silva (2010, p.88) as políticas públicas de assistência social, saúde e de previdência social:

"compõem o quadro das políticas públicas no que se refere às ações de proteção social implementadas pelo Estado. Elas complementam ações dessas últimas, pois estão diretamente ligadas às demandas causadas pelo sistema econômico capitalista, visando diminuir a desigualdade social entre as classes com maior e menor poder aquisitivo".

\section{A PSICOLOGIA DA SAÚDE E O SOFRIMENTO ÉTICO-POLÍTICO}

As proposições da Psicologia da Saúde em sua articulação com a Assistência Social, exigem saberes ligados a análise de demandas comunitárias. Sarriera, Freitas e Scarparo (2003, p.176) enfatizam que o profissional de psicologia que intente atuar neste campo necessita integrar as capacidades de "planejar e executar programas de ação comunitária, e a de identificar e problematizar temas relevantes à vida cotidiana". Deste modo, a compreensão apresentada para o adoecer, se expressaria no espaço relacional da comunidade, representaria uma condição de sofrimento ético-político e, conforme apontado por Sawaia (2004, p.102) "o sofrimento é a dor mediada pelas injustiças sociais".

A compreensão do sofrimento ético-político abrange o reconhecimento dos afetos presentes em contextos de exclusão, conforme destacado por Hinkel e Maheirie (2007). Os autores analisam os afetos envolvidos na música originada em comunidades pobres e, ao adotar o referencial da Psicologia Sócio-Histórica, discutem a amarração do sofrimento as condições materiais da vida. Este caminho também é trilhado por Duarte e Rumin (2008) que ofereciam acolhimento a famílias em situação de risco social. Já Brito (2005) buscou propiciar a ruptura do sofrimento ético-político e a conseqüente promoção da saúde por meio da capacitação para o trabalho e a inserção de populações jovens no mercado de trabalho. 
Neste último trabalho verifica-se o alargamento analítico em torno da noção do sofrimento ético-político e a urgência de posicionamento crítico em relação às políticas públicas que visam minorar a exclusão do mercado de trabalho. Tal posicionamento deve pautar-se pelo seguinte questionamento: "O que está em jogo é a organização dos trabalhadores e seu nível de consciência. A alternativa ao emprego é estratégia de sobrevivência ou forma superior de organização das relações de trabalho?" (Furtado, 2012 p.464)

Abordar as políticas públicas dirigidas ao trabalho não se restringe à inclusão em alguma modalidade produtiva (formal ou informal). Bucher (2003) já apontava este tendência ao discutir os rumos da profissão no início da década de 2000. Alcançando a extensão dos apontamentos de Furtado (2012) sobre "quais relações de trabalho", a Psicologia da Saúde move-se em direção a Saúde do Trabalhador.

\section{A SAÚDE DO TRABALHADOR E A PSICOLOGIA DA SAÚDE}

As intervenções que abordam a Qualidade de Vida no Trabalho (QVT) se apresentam na prática profissional do psicólogo guardando distância considerável das produções em saúde do trabalhador. Ao analisar empresas onde a QVT é alardeada como política organizacional verifica-se não haver reflexos positivos destas práticas sobre "as condições específicas de trabalho e de vida do empregado" (Tolfo e Piccinini, 2001 p.190). Ferreira, Alves e Tostes (2009) ao caracterizar e discutir as práticas em QVT em 10 órgãos públicos federais reafirmam a permanência das perspectivas expostas por Tolfo e Piccinini (2001) onde o trabalhador é a "variável de ajuste" do conjunto organizacional.

Em oposição às perspectivas da QVT, as práticas em Psicologia da Saúde orientadas à Saúde do Trabalhador proporcionam o questionamento da organização e das condições de trabalho, os limites de acesso aos serviços dirigidos a reabilitação de trabalhadores e serviços de previdência social. Representante da interdisciplinaridade na Psicologia, a saúde do trabalhador expõe claramente como determinantes de desgaste à saúde originam-se na organização e nas condições de trabalho. Assim, efetivamente são questionadas as relações de trabalho, conforme propõe Furtado (2012).

Sato, Lacaz e Bernardo (2006) posicionam as intervenções em saúde do trabalhador na história da Psicologia brasileira, a partir da década de 1980 com 
as pesquisas originadas pelo Departamento Intersindical de Estudos e Pesquisas de Saúde e dos Ambientes de Trabalho (DIESAT) e pelo surgimento dos primeiros programas de saúde do trabalhador ligados ao SUS. Conforme os autores, Psicologia e Saúde do Trabalhador experimentam:

"por um lado a forte presença de regulamentações legais e a conformação do SUS [que] deram nortes, constrangeram ou abriram possibilidades para a construção de um campo para a Psicologia; por outro, deve-se ressaltar que, também a abordagem psicológica influiu na definição de regulamentações legais previdenciárias" (Sato, Lacaz e Bernardo, 2006 p.286).

A tomada do trabalho como elemento pautado pelas relações da coletividade direciona a saúde do trabalhador às políticas públicas do Sistema Único de Saúde (SUS) e, por isso, observa-se "o trânsito entre as representações individuais e o caráter coletivo do trabalho" (Codo, 2006, p. 57). Este trânsito é apresentado nos marcos legais que estabelecem a Rede Nacional de Atenção Integral à Saúde do Trabalhador (RENAST) e a Política Nacional de Segurança e Saúde no Trabalho (PNSST). A RENAST prevê, na totalidade dos níveis de atenção do SUS, ações de promoção, prevenção, cura e reabilitação em saúde do trabalhador. Aos psicólogos que já atuam nos serviços do SUS, caberá o pressuposto de articular suas práticas em saúde à relação entre o homem e o trabalho. Na proposição do PNSST, as ações da Psicologia ligadas a Saúde do Trabalhador necessitarão reorientar seu processo formativo no ensino superior, promover pesquisas em saúde do trabalhador, fortalecer a vigilância de processos e agravos à saúde e estabelecer a adequação e ampliação das intervenções dirigidas à reabilitação física e psicossocial.

A formação de profissionais a partir de supostos da Psicologia da Saúde já apresenta um amplo referencial teórico e técnico. Isto pode ser observado nos congressos da Associação Brasileira de Psicologia da Saúde (ABPSA) e nas publicações científicas indexadas no Scielo, na BVS-Psi, na Redalyc e no DOAJ. Entretanto, a proposição do PNSST exigiria maior permeabilidade da Psicologia da Saúde aos conteúdos oriundos do campo Saúde do Trabalhador. Em congruência com as indicações de Jacques (2007), o estímulo aos processos formativos que preconizariam o reconhecimento do nexo causal entre atividade produtiva e adoecimento seria o instrumento para a execução da vigilância em saúde do trabalhador no SUS.

O conjunto de ações presentes no PNSST faz parte de um campo histórico de atuação da Psicologia da Saúde. Conforme ressalta Calatayud (2011) a 
Psicologia da Saúde em Cuba desenvolve-se no Instituto de Salud de los Trabajadores há 35 anos, constituindo referencias para o reconhecimento do nexo causal entre impacto à saúde e o trabalho. Almirall e Hernández (2010, p.67) pormenorizam estas ações cubanas da Psicologia em Saúde do Trabalhador: "evaluación de la carga psíquica, fatiga, estrés, burnout y mobbing, y su impacto en la salud y el rendimiento laboral".

Mesmo na produção cubana sobre Psicologia e Saúde do Trabalhador, a reabilitação ainda é rudimentar. No Brasil, isto decorre da fragilidade dos serviços de saúde, que contam com uma rede discreta de Centros de Referência em Saúde do Trabalhador (CEREST). Também contribui para a fragilidade da reabilitação o desmonte destas estruturas do Instituto Nacional de Seguro Social (INSS), mesmo havendo legislação que defina a execução deste serviço por este serviço público. A participação dos profissionais de Psicologia na reestruturação da reabilitação no INSS cumpriria a atribuição da integralidade dos serviços em saúde do trabalhador prestados pelos serviços públicos, de acordo com a normatização do PNSST (Brasil, 2011). Com esta participação estaria referenciado o cuidado integral, que historicamente desconsiderou a saúde mental nas práticas de reabilitação do INSS.

\section{CONSIDERAÇÕES FINAIS}

A observação das notas presentes neste texto, referentes aos marcos da Psicologia da Saúde no Brasil possibilita algumas reflexões. Pode-se iniciar tal esforço indicando a expansão das práticas formativas da Psicologia Hospitalar na atenção terciária, das Residências Profissionais e de outras práticas ligadas a Estratégia de Saúde da Família (atenção primária) em direção ao fortalecimento teórico e técnico da Psicologia da Saúde na atenção secundária. A ampliação das práticas de Psicologia na atenção secundária estruturaria uma rede de referência direcionada a integralidade do cuidado à saúde. Este princípio do SUS - a integralidade - é diretamente prejudicado com a fragmentação da atenção secundária.

O fortalecimento das políticas de Assistência Social indica o reconhecimento do sofrimento ético-político e articula-se as práticas de Psicologia de Saúde por preconizar a qualidade de vida como pressuposto que orienta as modalidades interventivas. Cabe a Psicologia da Saúde apropriar-se de práticas dirigidas a comunidade - tal como as metodologias participativas para a promoção da saúde 
(Silva, 2002) - e conectar-se ao corpo de saberes oriundos da Psicologia Comunitária - que se desenvolveu na América Latina - e da Psicologia Social (da Saúde e do Trabalho). Tanto a Psicologia Comunitária quanto a Psicologia Social ofertam experiências de práticas dirigidas ao fortalecimento da família, a promoção de direitos civis e de fomento a autonomia, conforme propõe o Conselho Federal de Psicologia ao orientar a prática profissional no Sistema Único de Assistência Social (CFP, 2011).

A participação da Psicologia da Saúde nas políticas públicas pode ser ampliado para além do SUS e do SUAS abrangendo o Ministério do Trabalho e Emprego e o Instituto Nacional de Seguro Social. Com o incentivo do Ministério do Trabalho seria possível constituir evidências e referências técnicas para as práticas em Psicologia da Saúde nos ambientes de trabalho. Vale ressaltar, que os determinantes de agravos à saúde mental oriundos dos processos produtivos já receberam atenção deste ministério nas edições especiais da Revista Brasileira de Saúde Ocupacional. A partir daqui caberia avaliar evidências de intervenções em saúde do trabalhador e divulgar experiências notórias que se estabeleceriam como referências de atuação em Psicologia da Saúde. Junto ao Instituto Nacional de Seguro Social, as práticas em Psicologia da Saúde envolveriam a reabilitação. Destaca-se que as ações de reabilitação atualmente desenvolvidas são pautadas em processos de ampliação da escolaridade e em intervenções reparadoras da funcionalidade do aparato corpóreo. São restritas as abordagens que envolvam o desenvolvimento da autonomia e nem mesmo há cuidados em saúde mental. Assim, seguir as proposições existentes no Programa Nacional de Segurança e Saúde no Trabalho (PNSST), exigiria reorientações das condutas dos Ministérios do Trabalho e da Previdência Social em direção as práticas em Psicologia da Saúde. Finaliza-se reafirmando o compromisso da Psicologia da Saúde com a participação no controle social orientado ao acompanhamento das políticas públicas e suas construções.

\section{REFERÊNCIAS}

Almirall, P.J.H; Hernández, J.S.R. (2010). Investigación científica en el Instituto Nacional de Salud de los trabajadores de Cuba (1998-2008). Algunas reflexiones y proyecciones. Revista Cubana de Salud y Trabajo, 11(1), 5970. 
Alves, R.R.N.; Alves, H.N.; Barboza, R.R.D.; Souto, W.M.S. (2010) The influence of religiosity on health. Ciência e Saúde Coletiva, 15(4), 2105-2111.

Associação Brasileira de Ensino em Psicologia (ABEP). (2006) A presença qualificada no SUS como um desafio para a Psicologia: Proposta da oficina nacional da ABEP. Ministério da Saúde/Ministério da Educação/Organização Panamericana de Saúde: Brasília.

Ávila, L. A. (2004). Grupo e corpo, no enfoque do modelo de Cambridge. Vínculo, $1(1), 23-29$.

Ávila, L. A. (2006). A secreta simetria: Grupo e corpo na compreensão psicanalítica. Revista SPAGESP, 7(1), 17-27.

Bernardes, A. G., \& Guareschi, N. M. F. (2010). Práticas psicológicas: enfrentamentos entre saúde pública e saúde coletiva. Estudos de Psicologia, 15(3), 269-276.

Bittencourt, R. A. A.; Mateus, M. L. F. (2006). Possibilidades de atuação do psicólogo no programa saúde da família: A experiência de Bonito-MS. Psicologia: Ciência e Profissão, 26(2), 328-343.

Boal, A. (1975). Teatro do Oprimido e outras Poéticas Políticas. Rio de Janeiro: Civilização Brasileira.

Brasil. (2006). Acolhimento nas práticas de produção de saúde. Brasília: Ministério da Saúde.

Brasil. (2011). Decreto no 7.602, de 7 de novembro de 2011. Presidência da República: Brasília.

Brito, A. J. R. (2005). Consórcio Social da Juventude de São Paulo: Tecendo considerações sobre as escolhas e as perspectivas de inserção no mundo do trabalho das juventudes. Imaginário, 11(11), 161-187.

Bucher, J. S. N. F. (2003). Psicologia da Saúde no contexto da saúde pública: Uma complexidade crescente. In: Yamamoto, O. H.; Gouveia, V. V. Construindo a psicologia brasileira: Desafios da ciência e prática psicológica. São Paulo: Casa do Psicólogo, p. 213-240.

Calatayud, F. M. (2011). La investigación en Psicología de la Salud en Cuba: Experiencias y potencialidades. Estudos de Psicologia, 16(1), 23-30.

Calvetti, P.U.; Muller, M.C.; Nunes, M. L. T. (2007). Psicologia da Saúde e Psicologia Positiva: perspectivas e desafios. Psicologia: Ciência e Profissão, 27(4), 706-717. 
Camargo-Borges, C.; Cardoso, C. L. (2005). A Psicologia e a estratégia saúde da família: Compondo saberes e fazeres. Psicologia \& Sociedade, 17(2), 26-32.

Castro, E.K.; Bornholdt, E. (2004). Psicologia da Saúde x Psicologia Hospitalar: definições e possibilidades de inserção Profissional. Psicologia: Ciência e Profissão, 24(3), 48-57.

Codo, W. (2006). Precisamos de trabalho, não de força de trabalho. In:

(Org.). Por uma Psicologia do Trabalho: Ensaios recolhidos. São Paulo: Casa do Psicólogo, p.61-74.

Conselho Federal de Psicologia. (2011). Como os psicólogos e as psicólogas podem contribuir para avançar o Sistema Único de Assistência Social (SUAS) - informações para gestores e gestoras. Brasília: CFP.

Costa, F. L.; Brandão, N. S. (2005). Abordagem Clinica no Contexto Comunitário: uma perspectiva integradora. Psicologia \& Sociedade, 17(2), 33-41.

Dimenstein, M. (2000). A cultura profissional do Psicólogo e o ideário individualista: Implicações para a prática no campo da assistência pública à saúde. Estudos de Psicologia, 5(1), 95-121.

Duarte, L. C. S.; Rumin, C. R. (2008). Práticas assistenciais e Psicologia da Saúde: Enlaces e proposições técnicas. Omnia Saúde, 5(supl.), 29-44.

Dunn, A.S.; Julian, T.; Formolo, L.R.; Green, B.N.; Chicoine, D.R. (2011). Preliminary analysis of posttraumatic stress disorder screening within specialty clinic setting for OIF/OEF veterans seeking care for neck or back pain. Journal of Rehabilitation Research \& Development, 48(5), 493-502.

Ferreira, M.C.; Alves, L.; Tostes, N. (2009). Gestão de qualidade de vida no trabalho (QVT) no serviço público federal: O descompasso entre problemas e práticas gerenciais. Psicologia: Teoria e Pesquisa, 25(3), 319-327.

Flórez-Alarcón, L. (2006). La Psicología de la Salud en Colombia. Universitas Psychologica, 5(3), 681-693.

Furtado. O. (2012). O trabalho na perspectiva das políticas públicas. In: JacóVilela, A.M.; Sato, L. Diálogos em Psicologia Social. Rio de Janeiro: Centro Edelstein de Pesquisa Social, p.462-474. 
Gascon, M.R.P.; Bonfim, M.C.; Pedroso, T.G.; Campos T.R.; Benute, G.R.G.; Aoki, V.; Takaoka, R.; Leão, R.O.; Lúcia, M.C.S.; Festa Neto, C. (2012). Avaliação psicológica de crianças com dermatite atópica por meio do teste das fábulas de Düss. Estudos Interdisciplinares em Psicologia, 3(2), 182195.

Gidron, Y. (2002). Psicologia da Saúde baseada na evidência: racional e suporte. Psicologia, Saúde e Doenças, Lisboa, 3(1), 03-10.

Gorayeb, R. (2010). Psicologia da Saúde no Brasil. Psicologia: Teoria e Pesquisa, 26(especial), 115-122.

Grilo, A. M.; Pedro, H. (2005). Contributos da Psicologia para as profissões da saúde. Psicologia, Saúde e Doenças, Lisboa, 6(1), 60-89.

Guareschi, N.M.F.; Dhein, G.; Reis, C.; Machry, D.S.; Bennemann, T. (2009). A formação em Psicologia e o profissional da Saúde para o SUS (Sistema Único de Saúde). Arquivos Brasileiros de Psicologia, 61(3), 35-45.

Guirado, M. (2004). Instituição e relações afetivas: O vínculo com o abandono. São Paulo, Casa do Psicólogo.

Hinkel, J.; Maheirie, K. (2007). RAP - Rimas Afetivas da Periferia: Reflexões na perspectiva sócio-histórica. Psicologia \& Sociedade, 19(esp. 2), 90-99.

Jacques, M. G. (2007). O nexo causal em saúde/doença mental no trabalho: Uma demanda para a psicologia. Psicologia \& Sociedade, 19(supl), 112-119.

Jin, S.G.; Xiang, B.; Yan, L.N.; Chen, Z.Y.; Yang, J.Y.; Xu, M.Q., Wang, W.T. (2012). Quality of life and psychological outcome of donors after living donor liver transplantation. World Journal of Gastroenterology, 18(2), 182187.

Jovchelovitch, S. (2008). Os contextos do saber: Representações, comunidade e cultura. Petrópolis: Vozes.

Käes, R. (2005). Os espaços psíquicos comuns e partilhados: Transmissão e negatividade. São Paulo: Casa do Psicólogo.

Miyazaki, M.; Cristina, O. S.; Domingos, N. A. M.; Valerio, N. I.; Santos, A. R. R.; Rosa, L. T. B. (2002). Psicologia da saúde: Extensão de serviços à comunidade, ensino e pesquisa. Psicologia USP, 13(1), 29-53.

Nery, M. P.; Costa, L. F. (2008). A pesquisa em psicologia clínica: Do indivíduo ao grupo. Estudos de Psicologia, 25(2), 241-250. 
Péres-Pareja, J.; Sesé, A.; González-ordi, H.; Palmer, A. (2010). Fibromyalgia and chronic pain: Are there discriminating patterns by using the Minnesota Multiphasic Personality Inventory-2 (MMPI-2)? International Journal of Clinical and Health Psychology, 10(1), 41-56.

Perez, D. K.; Constantino, E.P.; Silva, C.M.R. (2010). O psicólogo nas políticas públicas sociais: Reflexões sobre as práticas Psi. In: CONSTANTINO, E.P. Psicologia, Estado e Políticas Públicas. Assis: UNESP, p. 87-108.

Santos, E.; Kirschbaum, D. (2008). A trajetória histórica da visita domiciliária no Brasil: Uma revisão bibliográfica. Revista Eletrônica de Enfermagem, 10(1), 220-227.

Sarriera, J. C.; Freitas, M. De F. Q.; \& Scarparo, H. Para onde caminha a Psicologia (Social) Comunitária no Brasil? Um balanço a partir dos frutos da sua trajetória. In: Yamamoto, O.H.; Gouveia,V.V. (Org.). Construindo a Psicologia Brasileira: Desafios da ciência e prática psicológica. São Paulo: Casa do Psicólogo, 2003.

Sato, L.; Lacaz, F.A.C.; Bernardo, M.H. (2006). Psicologia e saúde do trabalhador: Práticas e investigações na saúde pública de São Paulo. Estudos de Psicologia, 11(3), 281-288.

Sawaia, B.B. (2004). As artimanhas da exclusão: Análise psicossocial e ética da desigualdade social. Petrópolis: Vozes.

Scarparo, H.B.K.; Guareschi, N.M.F. (2007). Psicologia social comunitária profissional. Psicologia e Sociedade, 19(supl.2), 100-108.

Sebastiani, R.W.; Maia, E.M.C. (2006). Análisis de la evolución de la producción científica presentada en eventos académicos de Psicología da la Salud en América Latina (1985-2003). Acta Colombina de Psicología, 9(1), 05-24.

Silva, R.C. (2002). Metodologias participativas para trabalhos de promoção de saúde e cidadania. São Paulo: Vetor.

Silveira, D.P.; Vieira, A.L.S. (2009). Saúde mental e atenção básica em saúde: Análise de uma experiência no nível local. Ciência e Saúde Coletiva, 14(1), 139-148.

Soares, T. C. (2005). A vida é mais forte do que as teorias: O psicólogo nos serviços de atenção primária à saúde. Psicologia Ciência e Profissão, 25(4), 590-601. 
Sousa, V.D.; Cury, V. E. (2009). Psicologia e atenção básica: vivências de estagiários na Estratégia de Saúde da Família. Ciência e Saúde Coletiva, 14(supl.1), 1429-1438.

Spink, M. J. P. (2007). Sobre a possibilidade de conciliação do ideal da integralidade nos cuidados à saúde e a cacofonia da demanda. Saúde e Sociedade, 16(1), 18-27.

Spink, M. J. P. (2003). Psicologia social e saúde: Práticas, saberes e sentidos. Petrópolis: Vozes.

Tolfo, S.R.; Piccinini, V.C. (2001). As melhores empresas para trabalhar no Brasil e a qualidade de vida no trabalho: Disjunções entre a teoria e a prática. Revista de Administração Contemporânea, 5(1), 165-193.

Contato: cassianorumin@fai.com.br

Recebido em: 01/04/2013

Revisado em: 05/05/2013

Aceito em: 23/06/2013 